\title{
Calidad de la Relación Universidad-Empresa en una Universidad Pública de Colombia
}

\author{
Silvana Correa-Henao ${ }^{1 *}$, Beatriz Londoño Giraldo ${ }^{2}$, Juan Fernando Tavera Mesías ${ }^{2}$
}

Resumen: El presente estudio tiene como objetivo identificar el papel de la función de marketing en los procesos de transferencia tecnológica universitaria, mediante la definición de hipótesis fundamentadas en el conocimiento científico del marketing y basadas en el caso de una universidad pública colombiana. Se ha diseñado un modelo de hipótesis que refleja la calidad de la relación, analizando constructos como satisfacción, confianza, compromiso y lealtad en la relación Universidad-Empresa, mediante un análisis confirmatorio. En los resultados, se evidencian los constructos más relevantes para gestionar y establecer relaciones de largo plazo entre la universidad analizada y las Empresas, lo que permite pasar de una transferencia tecnológica transaccional a una vista desde lo relacional.

Palabras Clave: Relación Universidad-Empresa; transferencia tecnológica universitaria; marketing relacional.

\begin{abstract}
Title: University-Industry Relationship Quality in a Public University of Colombia
The objective of this study is to identify the role of the marketing function in university technology transfer processes, through the definition of hypotheses based on the scientific knowledge of marketing and the bases in the case of a colombian public university. It has been designed a hypothesis model that reflects the quality of the relationship, analyzing constructs such as satisfaction, trust, commitment and loyalty in the University-Industry relationship, through a confirmatory analysis. In the results, the most relevant constructs for the management and the long-term relationships between the university and the companies are evidenced, which allows to pass from a technological transaction to a relationship view.
\end{abstract}

Keywords: University-Industry relationship; university technology transfer; relationship marketing.

Submitted: Jun $6^{\text {th }}, 2018 /$ Approved: August $19^{\text {th }}, 2018$

\section{Introducción}

El presente estudio desarrolla un análisis que contribuye al reconocimiento de la calidad de la relación entre la Universidad y la Empresa desde la perspectiva del marketing relacional, el cual, se basó en primer lugar, en una revisión de literatura, constituida con argumentos, conceptos y avances actualizados de la literatura académica, y en segundo lugar, en un análisis confirmatorio aplicado en el contexto de una universidad pública colombiana.

En la literatura se ha abordado la relación Universidad-Empresa desde la perspectiva de la innovación, encontrando que un factor determinante para que se dieran de forma cercana y organizada fue principalmente el de las guerras, ya que allí se evidenció la importancia de establecer una sinergia entre las universidades como generadoras de conocimiento, la industria como desarrolladora de tecnología y recursos, y el Estado como generador de políticas y ente regulador de las relaciones (Sábato y Botana, 1968; Zacarías y Martín, 2011).

Diferentes autores han descrito esta relación bajo diferentes modelos (Bozeman, 2000; Cook, Uranga, y Etxebarria, 1997; Leydesdorff, 2012; Leydesdorff y Etzkowitz, 1998; Lundvall, 1997; Sábato y Botana, 1968), dentro de los que se resaltan el triángulo de Sábato (Sábato y Botana, 1968) y el modelo de la triple hélice (Leydesdorff y Etzkowitz, 1998) por su gran acogida.
A partir de la ley Bayh Dole en los 80's hubo se evidencia un incremento en los estudios sobre la relación Universidad-Empresa, fortaleciéndose aún más en los 90 's, interactuando usualmente mediante las patentes, acuerdos de licencia e investigación conjunta (Barcelo, España, y Prieto, 2012). Para facilitar estas relaciones, se crearon las oficinas de transferencia tecnológica que se encargaran del proceso de intermediación para las negociaciones entre la Universidad y la Industria (Martinelli, Meyer, y von Tunzelmann, 2008).

Existen numerosos estudios sobre la relación Universidad-Empresa abordados desde el punto de vista de la transferencia tecnológica, sin embargo la interacción entre estos actores sigue presentando fallas (Cyert y Goodman, 1997; Lee, 2000), por lo que algunos autores han tratado de llegar a una solución abordando el problema desde diferentes perspectivas, como es el caso del enfoque relacional basado en el marketing (Plewa, Quester, y Baaken, 2005). Sin embargo, los aportes en este tema aún son limitados (Rosendo Ríos, 2013).

En Colombia, en materia de transferencia tecnológica aún se está en proceso de estructuración apoyado por el Sistema Nacional de Ciencia, Tecnología e Innovación, SNCTI que se crea a través del Departamento Administrativo de Ciencia, Tecnología e Innovación, Colciencias, con el fin de "promover y consolidar mecanismos de inversión en las actividades de investigación y desarrollo y la formación del capital humano en CTI como instrumentos determinantes del desarrollo económico, social y ambiental" (DNP, 2009).

(1) Department of Administrative Sciences, Instituto Tecnológico Metropolitano, Medellín, Colombia.

(2) Department of Administration, Universidad de Antioquia, Medellín, Colombia.

*Corresponding author: silvanacorrea@itm.edu.co

ISSN: 0718-2724. (http://jotmi.org)

Journal of Technology Management \& Innovation @ Universidad Alberto Hurtado, Facultad de Economía y Negocios. 
El SNCTI permite diferentes procesos de vinculación y actividades conjuntas entre las universidades y las empresas, los cuales, se dan en varias ocasiones, gracias a las convocatorias y diferentes iniciativas que el Estado hace para propiciar la transferencia de conocimiento, generando su impacto en el creciente trabajo entre la academia y la industria (Bozeman y Gaughan, 2007), aunque el escenario ideal sería que las Empresas incrementen su compromiso de invertir en generar nuevos desarrollos e investigaciones conjuntas con las Universidades, sin esperar la intervención del Estado.

Es por esto, que este estudio pretende realizar un acercamiento de tipo exploratorio y descriptivo para entender las relaciones Universidad-Empresa desde el punto de vista relacional, identificando el papel de la función del marketing en los procesos de transferencia tecnológica universitaria, mediante la definición de hipótesis fundamentadas en el conocimiento científico del marketing, y basadas en el contexto de una universidad pública colombiana.

\section{Marco teórico y desarrollo de hipótesis}

La literatura sobre transferencia tecnológica en el marco de la relación Universidad-Empresa se ha enfocado principalmente desde un punto de vista transaccional más que de uno relacional (Guerin, 1999; Plewa et al., 2007), lo que podría deberse a la tradicional perspectiva de hacer énfasis en el rol de las Universidades que solo transfieren sus resultados de investigación a otros actores como la sociedad y la empresa (Azagra-Caro, Gutiérrez-Gracia, y Fernández-de-Lucio, 2006; Castro, Cortés, Gelench, y Costa, 2005; Gunasekara, 2005; Necoechea-Mondragón, Pineda-Domínguez, y Soto-Flores, 2013).

Sin embargo, la relación Universidad-Empresa toma diversas formas, como es el caso de actividades en conjunto de $\mathrm{I}+\mathrm{D}$, teniendo una relación mucho más estrecha que la tradicional generada por la transferencia de resultados de investigación y comercialización (Perkmann y Walsh, 2007). En el contexto de esta relación, la universidad y la empresa crean una red organizacional colaborativa donde ambos actores realizan tareas en conjunto con objetivos diferentes, pero con una alta dependencia el uno del otro para lograr los resultados esperados, lo que implica la necesidad de un enfoque que ayude a gestionar estas relaciones cada vez más complejas (Mora-Valentin, Montoro-Sanchez, y Guerras-Martin, 2004; Perkmann y Walsh, 2007; Plewa et al., 2013).

Por lo anterior, es claro que el papel del marketing en la transferencia tecnológica es crucial, permitiendo a las universidades, además de la docencia y la investigación, jugar un rol activo en la sociedad mediante el establecimiento de relaciones con las empresas, ayudando a reducir la brecha entre la comunidad académica y empresarial (Frasquet et al., 2011; Lantos, 1994).

Desde el marketing se ha abordado la relación Universidad-Empresa desde la perspectiva del marketing relacional (Frasquet et al., 2011; Helgesen, 2008; Marzo-Navarro, Pedraja-Iglesias, y Rivera-Torres, 2009; Plewa et al., 2005), conocida como el proceso de identificar, establecer, mantener, mejorar, y cuando sea necesario terminar las relaciones con los clientes y otros stakeholders, de forma rentable, de tal manera que se cumplan los objetivos de las partes interesadas (Grönroos, 1997), ya que lo que se busca es definir qué factores son determinantes para generar relaciones duraderas entre estos actores. El marketing relacional ha representado un cambio significativo en la concepción del marketing desde un punto de vista meramente transaccional a uno enfocado en establecer relaciones de largo plazo (Lee, 2000; Mora-Valentín et al., 2004; Plewa et al., 2007), mismo cambio que se espera se genere en la relación Universidad-Empresa.

Siguiendo la perspectiva del marketing relacional, se identificaron cinco constructos que pueden ser útiles para explicar la relación Universidad-Empresa con un enfoque académico y profesional, como son la satisfacción, la confianza, el compromiso y la lealtad, los cuales influyen en la construcción de relaciones duraderas (Chenet, Dagger, y O'Sullivan, 2010; Gil-Saura, Frasquet-Deltoro, y Cervera-Taulet, 2009; Huntley, 2006).

En primer lugar, el valor percibido es definido por como la evaluación global por parte del consumidor de la utilidad de un producto, basada en la percepción de lo que se recibe y de lo que se entrega (Zeitha$\mathrm{ml}, 1988$ ). Así mismo, se define como todos los factores cualitativos y cuantitativos, subjetivos y objetivos inmersos en todo el proceso de compra (Schechter, 1984).

En segundo lugar, la satisfacción es considerada como la evaluación de las expectativas percibidas sobre un producto o servicio y la realidad (Oliver, 1999)

En tercer lugar, la confianza es vista como una creencia, sentimiento, o la expectativa sobre la confiabilidad de un compañero de intercambio que resulta de la experiencia de la pareja, la fiabilidad o la intencionalidad (Blau, 1964; Moorman, Zaltman, y Deshpande, 1992; Pruitt, 1981; Rotter, 1967).

En cuarto lugar, el compromiso se concibe como la intención de establecer y mantener relaciones de largo plazo (Moorman et al., 1992). Esta intención es demostrada con promesas implícitas o explícitas de beneficio para las partes involucradas, adicionalmente, es necesario hacer sacrificios para obtener dichos beneficios (Marzo-Navarro et al., 2009).

En quinto lugar, la lealtad es vista como la cantidad de re-compras que se hace a la misma empresa, considerando solo esa oferta y no haciendo ninguna búsqueda relacionada con la misma (Newman y Werbel, 1973; Oliver, 1999).

Con la definición de las hipótesis, se busca evidenciar desde la literatura del marketing relacional cómo se comportan los constructos planteados en la relación Universidad-Empresa, analizando el caso específico de la Universidad de Antioquia.

En la literatura del marketing, la satisfacción es considerada como constructo influenciador de la confianza (Oliver, 1999; Zeithaml, Berry, y Parasuraman, 1996), ya que un cliente puede generar confianza tras una acumulación de satisfacción en la experiencia del servicio. 
Por otro lado, diferentes autores toman la satisfacción como constructo influenciador de la lealtad (Gil-Saura et al., 2009; Jones y Sasser, 1998; Morgan y Hunt, 1994; Oliver, 1999), ya que la lealtad sin satisfacción difícilmente se da.

H1: La satisfacción influye positivamente sobre la confianza en la relación U-E

H2: La satisfacción influye positivamente sobre la lealtad en la relación U-E

Del mismo modo, se ha abordado la confianza como influenciador del compromiso (Achrol, 1991; Hrebiniak, 1974; McDonald, 1981; Moorman et al., 1992; Morgan y Hunt, 1994; Rotter, 1967), ya que la confianza es un factor importante para quienes quieren llegar al compromiso (Hrebiniak, 1974). Existe una relación directa entre la confianza y la lealtad (Umar y Bahrun, 2017; Setyawati y Raharja, 2018), sin embargo, algunos autores como Gil, Frasquet, y Cervera, (2009); Tian et al., (2008) encontraron relación significativa por medio de un efecto indirecto a través del compromiso.

H3: La confianza influye positivamente sobre el compromiso en la relación $\mathrm{U}-\mathrm{E}$

H4: La confianza influye positivamente sobre la lealtad en la relación U-E

Adicionalmente, el compromiso en la literatura ha sido identificado como influenciador de la lealtad (Moorman et al., 1992; Morgan y Hunt, 1994; Ulaga y Eggert, 2006), ya que en una relación ante un alto grado de compromiso, es muy posible generar lealtad.

H5: El compromiso influye positivamente sobre la lealtad en la relación U-E

Estas hipótesis se ven representadas en la Figura 1., con el fin de evidenciar las relaciones de forma más clara.
Figura 1. Modelo Conceptual e Hipótesis. Software SmatPLS

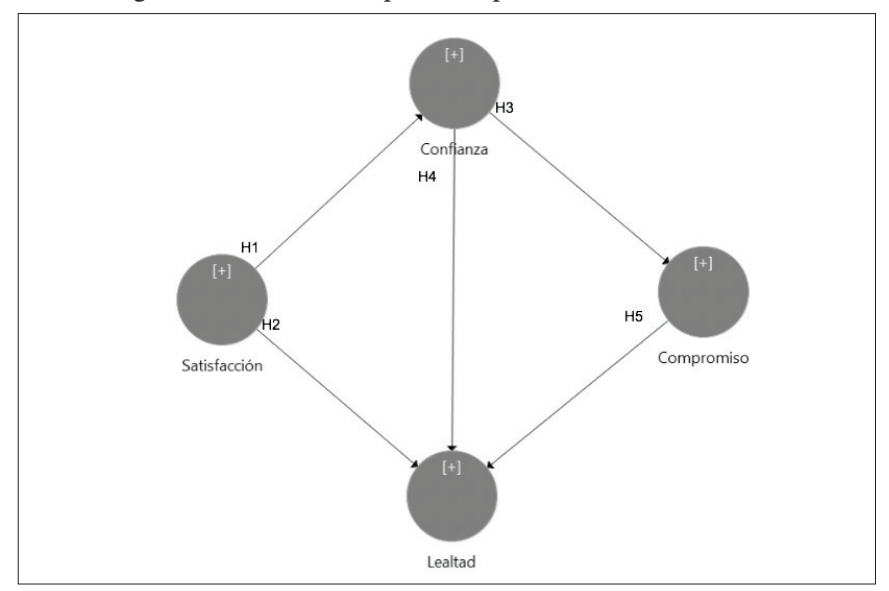

\section{Metodología}

Este estudio propone un modelo conceptual basado en los cuatro constructos que conforman la calidad de la relación en el contexto de una Universidad pública colombiana, la cual ha estado estructurando su proceso de transferencia tecnológica hace aproximadamente 14 años, buscando siempre el mejoramiento de la relación con las empresas.

Para el trabajo de campo en concordancia con los objetivos y las proposiciones planteadas en el modelo, se logró el análisis de la relación Universidad - Empresa en perspectiva de la calidad de la relación, esto para el caso puntual de los clientes que ha tenido la Universidad en los últimos 8 años.

Para este caso, se realizó una fase de análisis exploratorio compuesto por tres etapas, en la primera se hizo una revisión de literatura exhaustiva para dar cuenta de los antecedentes y estudios previos desarrollados que permitieron la identificación de los constructos adecuados para el planteamiento del modelo. En la Tabla 1 se evidencian los constructos así como las escalas utilizadas para el proceso de medición, las cuales fueron adecuadas al contexto y al objeto de estudio.

Tabla 1. Escalas de medición. Elaboración propia

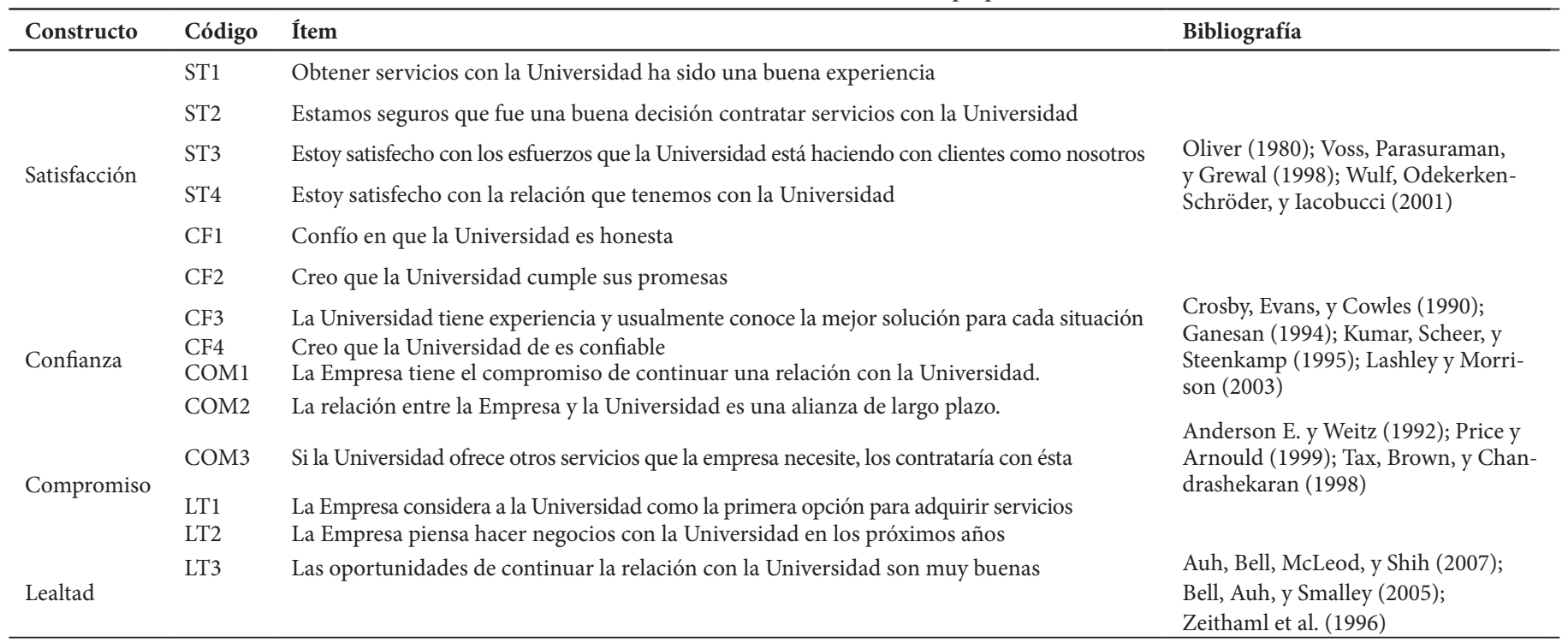


En la segunda etapa, se definió el tamaño de la muestra teniendo en cuenta que debían cumplir criterios de ser clientes de la Universidad de Antioquia, haber contratado servicios con la Universidad de Antioquia desde el 2010 hasta la fecha, teniendo como resultado una población finita (Scheaffer, Mendenhall, y Ott, 2007) de 143 empresas, de la cual con un nivel de confianza del 95\% se estima una muestra de mínimo 57 empresas para ser representativa.
Además, se construyó una muestra no probabilística a conveniencia de los clientes que contrataron servicios con la Universidad de Antioquia desde el 2010, enviando invitación a participar en una encuesta online a las 143 empresas y obteniendo respuesta de 61 lo que la hace representativa según las condiciones dadas. En la tabla 2 se evidencia la ficha técnica del estudio.

Tabla 2. Ficha Técnica de Trabajo de Campo. Elaboración propia

\begin{tabular}{ll}
\hline $\begin{array}{l}\text { Lugar } \\
\text { Tamaño de la muestra }\end{array}$ & $\begin{array}{l}\text { Medellín- Antioquia- Colombia } \\
\text { Unidad de } \\
\text { muestreo }\end{array}$ \\
$\begin{array}{l}\text { Método de la recolección } \\
\text { Escala }\end{array}$ & $\begin{array}{l}\text { Correctores y responsables de los procesos de relacionamiento para actividades de ciencia, tecnología e innovación } \\
\text { Instrumento delénica y visitas personales. }\end{array}$ \\
Método de análisis & Likert \\
\hline
\end{tabular}

Por último, se definieron hipótesis con los constructos identificados en la revisión de literatura en la relación Universidad-Empresa, para el caso puntual de la Universidad en estudio, realizando un

\section{Resultados}

Tras comprobar mediante la prueba Shapiro Wilks, ideal para muestras pequeñas (Razali y Wah, 2011; Shapiro y Wilk, 1965), que los datos no se comportan de acuerdo a una distribución normal, se procedió al análisis mediante el bootstrapping utilizando la muestra de análisis descriptivo, para dar una explicación al fenómeno mediante el uso de instrumentos de medición estructurados aplicados a la muestra definida.

61 empresas y las respuestas proporcionadas para cada constructo se robustece la información para proceder a correr el modelo y posteriormente realizar el análisis factorial confirmatorio y de ecuaciones estructurales propuesto en la Figura 1 utilizando Smart- PLS 3.0. A partir de esto se determina la validez y la fiabilidad del modelo.

Tabla 3. Cargas Externas. Elaboración propia

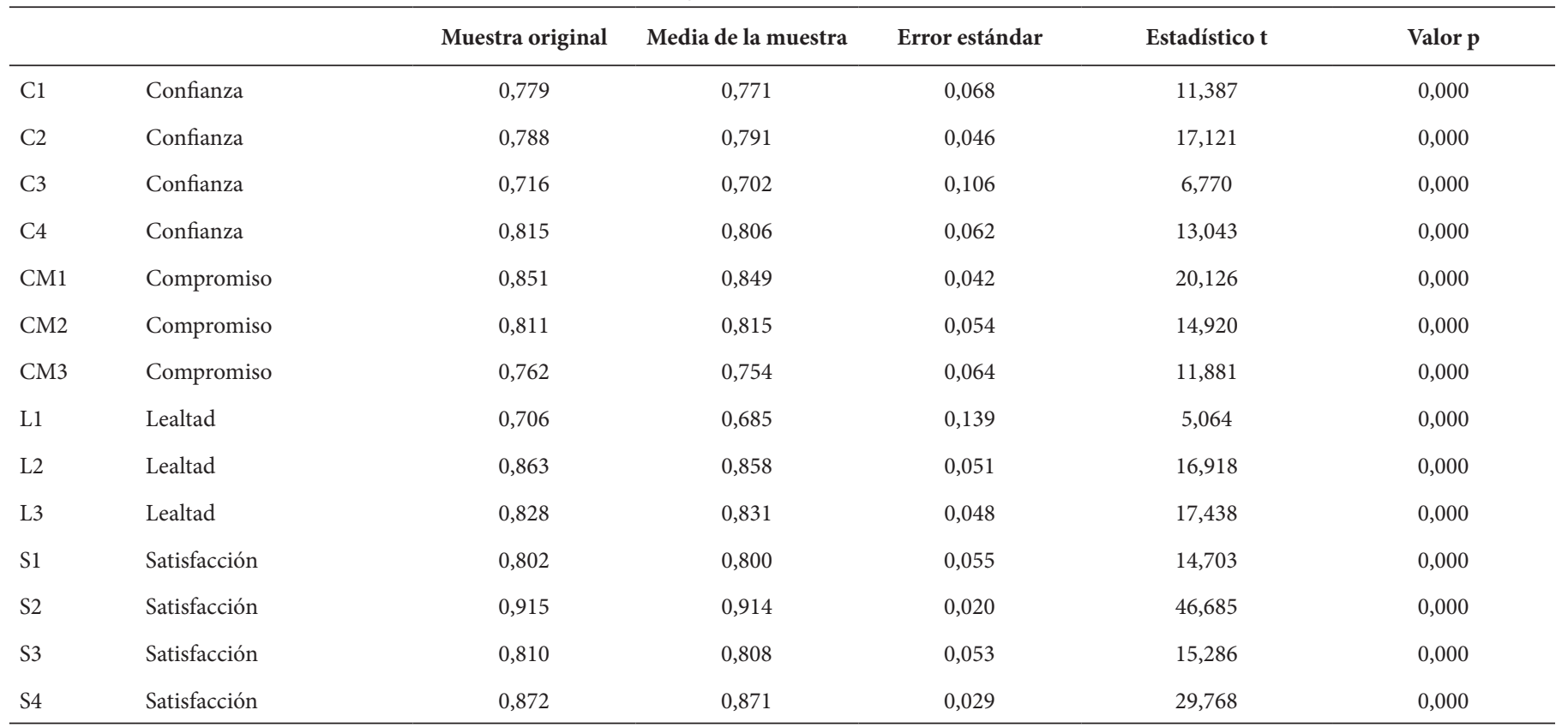


Los resultados de la tabla 3 evidencian la fiabilidad de la escala, lo que indica que las variables son el reflejo de que el constructo existe; los cuatro constructos muestran una fuerza alta hacia la variable y una menor de la variable al error; para todos los casos las cargas son superiores a 0,6 y los valores de p inferiores a 0,05, evidenciando la consistencia entre las variables.

Tabla 4. Alpha de Crombach

\begin{tabular}{llll}
\hline & Media & Desviación Estándar & Alpha de Crombach \\
\hline Confianza & 4,160 & 2,387 & 0,778 \\
Compromiso & 4,071 & 2,050 & 0,734 \\
Lealtad & 3,814 & 1,812 & 0,723 \\
Satisfacción & 3,922 & 2,884 & 0,872 \\
\hline
\end{tabular}

La tabla anterior confirma la fiabilidad a través del Alpha de Crombach, que en este caso son mayores a 0,7 (Nunnally y Bernstein, 1994). Así mismo, se muestra la media y la desviación estándar de cada uno de los constructos.

Por otro lado, de acuerdo con Hair et al. (2016), para validar los estudios en PLS, es necesario adoptar las siguientes etapas para el análisis del modelo: I) Instrumento de medida para los constructos: evaluando la consistencia interna, fiabilidad, validez convergente y divergente; II) Medida del modelo estructural: Evaluando los coeficientes de determinación y la significancia de las relaciones estructurales. En la tabla 5, se evidencian los criterios mínimos para analizar el modelo construido.

Tabla 5. Criterios mínimos establecidos para indicadores PLS-SEM (Hair, et al. 2016)

\begin{tabular}{|c|c|c|}
\hline Etapas de Validación & Indicadores & Criterios Mínimos \\
\hline \multirow[t]{5}{*}{$\begin{array}{l}\text { Instrumento de medida para } \\
\text { constructos }\end{array}$} & Consistencia Interna: Alpha de Cronbach (CA) & $=>0,7$ (Nunnaly y Bernstein, 1994). \\
\hline & Fiabilidad: Fiabilidad Compuesta (CR) & $=>0,7$ (Fornell y Larcker, 1981). \\
\hline & Validez Convergente: Varianza Extraída (AVE) & $=>0,5$ (Fornell y Larcker, 1981). \\
\hline & Validez Convergente: Tamaño de cargas y significancia & $=>0,6($ Bagozzi and Yi, 1988) y $\mathrm{p}<0,001 ; \mathrm{p}<0,05 ; \mathrm{p}<0,01$. \\
\hline & Validez Discriminante: Heterotrait-Monotrait (HT/MT) & $=<0,9($ Henseler et al.,2014) \\
\hline \multirow[t]{2}{*}{ Modelo Estructural } & Coeficiente de determinación (Valor $\mathrm{R}^{2}$ ) & $\begin{array}{l}=>0,75 y=>0,51 \text { relevante, }=<0,50 \\
y=>0,26, \text { moderado, } y<0,25 \\
\text { débil (Hair et al., 2013) }\end{array}$ \\
\hline & Significancia de las relaciones estructurales & $\mathrm{p}<0,001$ \\
\hline
\end{tabular}

A continuación, se presentan los resultados para analizar el modelo

Tabla 6. Fiabilidad y validez convergente para constructos

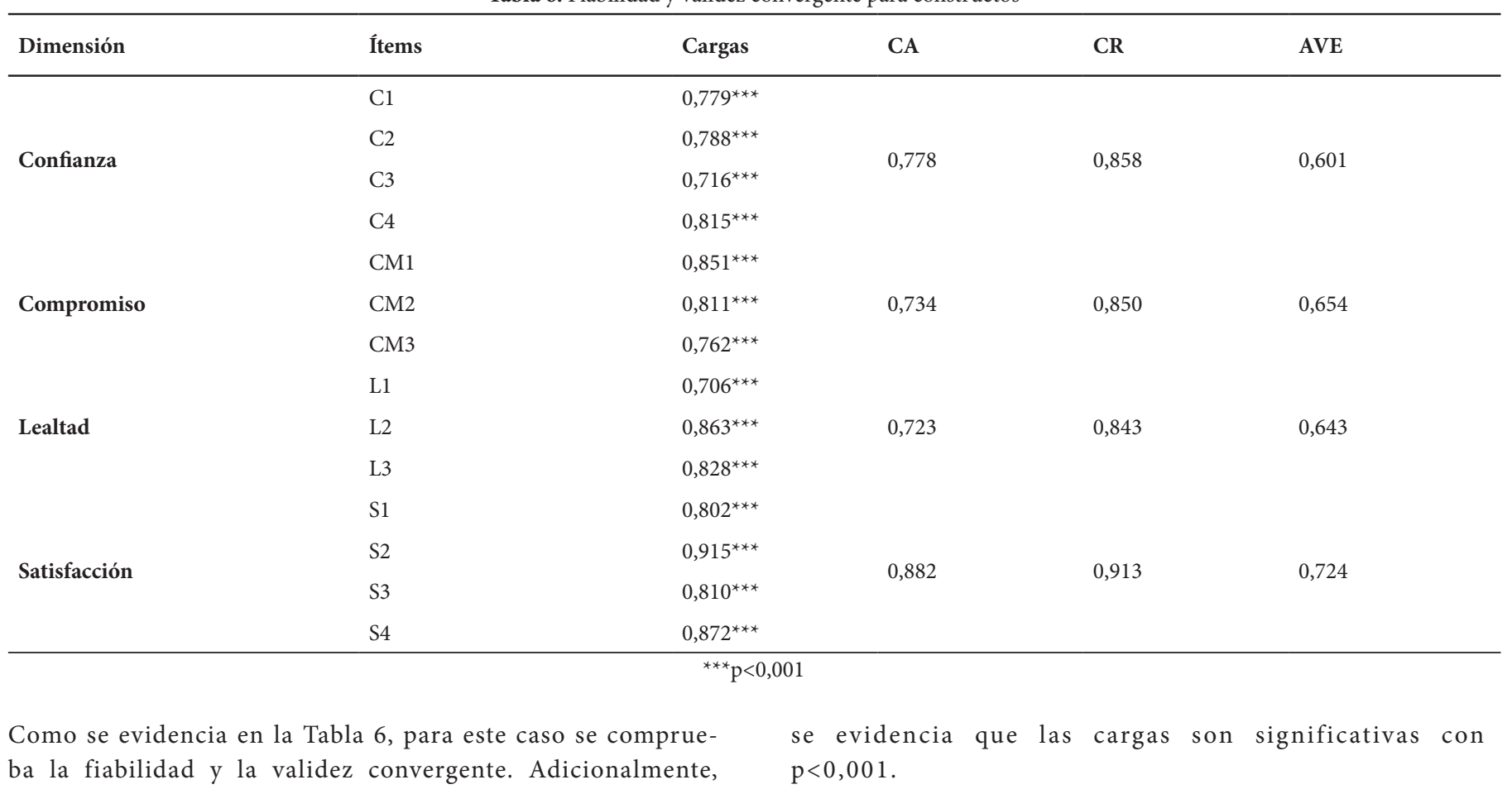


Tabla 7. Validez Discriminante de los constructos

\begin{tabular}{lllll}
\hline & CF & CM & LT & ST \\
\hline Confianza & 0,775 & & & \\
Compromiso & 0,486 & 0,809 & & \\
Lealtad & 0,558 & 0,597 & 0,802 & \\
Satisfacción & 0,697 & 0,364 & 0,434 & 0,851 \\
\hline
\end{tabular}

Como se muestra en la Tabla 7, el modelo presenta validez discriminante, mostrando en la diagonal el cuadrado de la varianza extraída (AVE) (Chin, 1998), calculada con el software SmartPLS.

Para evaluar la capacidad predictiva del modelo estructural, se utiliza el $\mathrm{R}^{2}$, el cual indica qué parte de la varianza de las variables dependientes son explicadas por las variables latentes influyentes (Aldás, 2017). Los resultados arrojados por el software SmartPLS muestran que los constructos confianza $\left(R^{2}=0,486\right)$ y lealtad $\left(R^{2}=0,452\right)$ se evidencian como indicadores moderados, en contraste con el compromiso $\left(\mathrm{R}^{2}=0,236\right)$.
Tabla 8. Contraste de hipótesis

\begin{tabular}{lllll}
\hline $\begin{array}{l}\text { Hipó- } \\
\text { tesis }\end{array}$ & Descripción & $\begin{array}{l}\text { Coeficiente } \\
\text { de trayec- } \\
\text { toria }\end{array}$ & Valor t & $\begin{array}{l}\text { Confirma- } \\
\text { ción }\end{array}$ \\
\hline H1 & Satisfacción-> Confianza & $0,697^{* * *}$ & 10,647 & SI \\
H2 & Satisfacción $->$ Lealtad & 0,067 & 0,367 & NO \\
H3 & Confianza $->$ Compromiso & $0,486^{* * *}$ & 4,390 & SI \\
H4 & Confianza-> Lealtad & 0,305 & 1,841 & NO \\
H5 & Compromiso $->$ Lealtad & $0,424^{* * *}$ & 3,911 & SI \\
\hline
\end{tabular}

Indicador de Significancia: $\mathrm{p}<0,001^{* * *}$

Figura 2. Resultados del modelo estructural

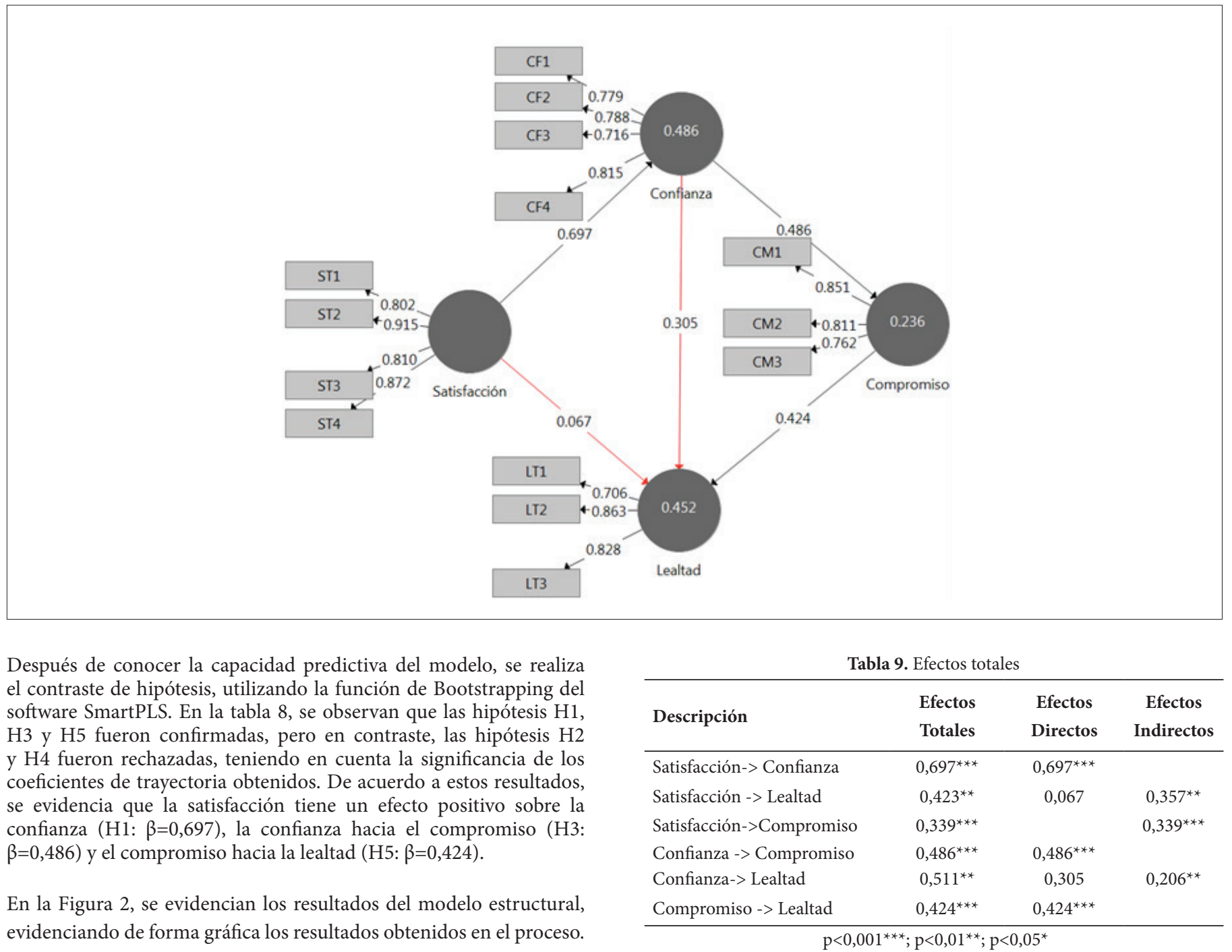


En la Tabla 9, se pueden evidenciar los efectos totales, los cuales permiten demostrar la existencia de relaciones entre todos los constructos. A pesar que de forma directa ni la satisfacción, ni la confianza presentan efectos directos sobre la lealtad, se evidencia que de forma indirecta presentan efectos de forma significativa con un $\mathrm{p}<0,01$.

\section{Discusión y Conclusiones}

El presente estudio contribuye a la literatura del marketing, evidenciando la importancia de establecer relaciones entre la Universidad y la Empresa mostrando particularmente cómo se comportan los factores principales de esta relación en el caso de una universidad pública colombiana para sus procesos de transferencia tecnológica.

Se construyó un marco teórico sobre la relación Universidad-Empresa vista desde el marketing relacional, y se propusieron hipótesis que identifican las relaciones existentes entre los constructos estudiados, validándolo mediante un análisis confirmatorio con base en una muestra empírica de datos, obtenida a partir de encuestas estructuradas enviadas a empresas que han adquirido servicios en la Universidad de Antioquia desde el 2010. Tras el análisis de información se pudo evidenciar que las hipótesis son aceptadas para el contexto de la Universidad de Antioquia en su relación con las empresas.

Los resultados evidenciaron que en la calidad de la relación Universidad-Empresa en el contexto analizado, el principal antecedente de la Lealtad de forma directa es el compromiso $\left(\beta=0,424^{* * *}\right)$, lo que se encuentra muy acorde con la literatura (Moorman et al., 1992; Morgan y Hunt, 1994; Ulaga y Eggert, 2006). Esto implica que las Universidades deben tratar de construir relaciones sólidas y generar un alto grado de compromiso con las Empresas para lograr relaciones de largo plazo.

Así mismo, se encontró que el principal antecedente de la lealtad teniendo en cuenta tanto los efectos directos $(\beta=0,305 ; \mathrm{p}=0,066)$, como efectos indirectos $(\beta=0,206 ; p=0,001)$ es la confianza $(\beta=0,511$; $\mathrm{p}=0,001$ ), ya que a través del compromiso es que se logra maximizar su efecto hacia la lealtad, esto en concordancia con autores como Tian et al., (2008); Gil, S. I, Frasquet, D. M. y Cervera, T. A. (2009) que encontraron resultados similares en sus estudios. Esto implica que las Universidades no solo deben trabajar en generar confianza entre las Empresas, deben llevarlo más allá, hacia un compromiso que logre convertirse en relaciones de lealtad en el largo plazo.

Algo similar ocurre con la satisfacción, la cual fue una relación que no dio significativa en sus efectos directos $(\beta=0,067 ; p=0,713)$ hacia la lealtad, pero teniendo en cuenta sus efectos indirectos $(\beta=0,357 ; p=0,004)$ mediada por la confianza y el compromiso, se puede llegar a influir positivamente en la lealtad $(\beta=0,423 ; p=0,001)$, lo cual es coherente con resultados encontrados en estudios como los de Hennig-Thurau, T., y Klee, A. (1997); Bowen, J. y Chen, S., (2001) ; Chiou, J. y Droge, C., (2006). Esto implica que las Universidades en una relación de largo plazo deben buscar más que satisfacer en un momento en el tiempo, deben lograr generar confianza y compromiso para llegar a la lealtad.
Todo esto permite concluir que es posible abordar la transferencia tecnológica desde el marketing relacional, generando mayor valor al evidenciar los factores más relevantes para la generación de relaciones de largo plazo con las empresas, permitiendo crear espacios de intercambios de valor que beneficien las partes y a la sociedad en general.

Este estudio tuvo limitaciones en su recolección de datos, ya que fue aplicado para el caso de una universidad pública en específico, lo que no permitiría generalizar los resultados para las Universidades Colombianas. Sin embargo, es un aporte que da cuenta de la importancia de profundizar en la investigación de este tema para lograr generar aportes más significativos para el comportamiento de la relación Universidad-Empresa en general visto desde el marketing relacional.

\section{Referencias}

Achrol, R. S. (1991). Evolution of the marketing organization: new forms for turbulent environments. the Journal of Marketing, 77-93. doi:10.2307/1251958.

Aldás, J. (2017). Modelización estructural con PLS-SEM: Constructos de segundo orden, Valencia (Spain), Universitat de València-Ivie.

Anderson, E., \& Weitz, B. (1992). The use of pledges to build and sustain commitment in distribution channels. Journal of marketing research, 18-34. doi:10.2307/3172490.

Auh, S., Bell, S. J., McLeod, C. S., \& Shih, E. (2007). Co-production and customer loyalty in financial services. Journal of retailing, 83(3), 359-370. doi:10.1016/j.jretai.2007.03.001.

Azagra-Caro, J. M., Archontakis, F., Gutiérrez-Gracia, A., \& Fernández-de-Lucio, I. (2006). Faculty support for the objectives of university-industry relations versus degree of R\&D cooperation: The importance of regional absorptive capacity. Research Policy, 35(1), 37-55. doi:10.1016/j.respol.2005.08.007.

Bagozzi, R. P., \& Yi, Y. (1988). On the evaluation of structural equation models. Journal of the academy of marketing science, 16(1), 74-94. doi:10.1177/009207038801600107.

Barcelo, R. A. V., España, J. L. N., \& Prieto, H. M. P. (2012). Motivaciones de los investigadores académicos en Colombia, para generar y transferir conocimiento al sector productivo usando análisis de correlación canónica. Estudios Gerenciales, 28(124), 125-139. doi:10.1016/ s0123-5923(12)70219-x.

Bell, S. J., Auh, S., \& Smalley, K. (2005). Customer relationship dynamics: service quality and customer loyalty in the context of varying levels of customer expertise and switching costs. Journal of the Academy of Marketing Science, 33(2), 169-183. doi:10.1177/0092070304269111.

Blau, P. M. (1964). Exchange and power in social life, New York: Wiley, $352 \mathrm{p}$. 
Bowen, J. T., \& Chen, S. L. (2001). The relationship between customer loyalty and customer satisfaction. International journal of contemporary hospitality management, 13(5), 213-217. doi:10.1108/09596110110395893.

Bozeman, B. (2000). Technology transfer and public policy: a review of research and theory. Research Policy, 29(4), 627-655. doi:10.1016/ s0048-7333(99)00093-1.

Bozeman, B., \& Gaughan, M. (2007). Impacts of grants and contracts on academic researchers' interactions with industry. Research policy, 36(5), 694-707. doi:10.1016/j.respol.2007.01.007.

Castro, E., Cortés, A., Gelench, M., \& Costa, C. (2005). Una aproximacion al análisis de impacto de las universidades en su entorno a través de un estudio de las actividades de las OTRI universitarias españolas. Altec.

Chin, W. W. (1998). The partial least squares approach to structural equation modeling. Modern methods for business research, (pp. 295336). NJ: Lawrence Erlbaum Associates.

Cook, P., Uranga, M. G., \& Etxebarria, G. (1997). Regional innovation systems: institutional and organizational dimensions. Research Policy, 26(4), 475-491. doi:10.1016/s0048-7333(97)00025-5.

Crosby, L. A., Evans, K. R., \& Cowles, D. (1990). Relationship quality in services selling: an interpersonal influence perspective. Journal of Marketing, 54(3), 68-81. doi:10.2307/1251817.

Cyert, R. M., \& Goodman, P. S. (1997). Creating effective universityindustry alliances: An organizational learning perspective. Organizational dynamics, 25(4), 45-57. doi:10.1016/s0090-2616(97)90036-x.

Chenet, P., Dagger, T. S., \& O’Sullivan, D. (2010). Service quality, trust, commitment and service differentiation in business relationships. Journal of Services Marketing, 24(5), 336-346. doi:10.1108/08876041011060440.

Chiou, J. S., \& Droge, C. (2006). Service quality, trust, specific asset investment, and expertise: Direct and indirect effects in a satisfactionloyalty framework. Journal of the academy of marketing science, 34(4), 613-627. doi:10.1177/0092070306286934.

DNP, Departamento Nacional de Planeación. (2009). Conpes 3582. Recuperado de http://www.dnp.gov.co/Portals/0/archivos/documentos/Subdireccion/Conpes/ 3582.pdf.

Eggert, A., Ulaga, W., \& Schultz, F. (2006). Value creation in the relationship life cycle: A quasi-longitudinal analysis. Industrial Marketing Management, 35(1), 20-27. doi:10.1016/j.indmarman.2005.07.003.

Frasquet, M., Calderón, H., \& Cervera, A. (2011). University-industry collaboration from a relationship marketing perspective: an empirical analysis in a Spanish University. Higher Education, 64(1), 85-98. doi:10.1007/s10734-011-9482-3.
Fornell, C., \& Larcker, D. F. (1981). Evaluating structural equation models with unobservable variables and measurement error. Journal of marketing research, 18(1), 39-50. doi:10.2307/3151312.

Ganesan, S. (1994). Determinants of Long-Term Orientation in Buyer-Seller Relationships. Journal of Marketing, 58(2), 1-19. doi:10.2307/1252265

Gil-Saura, I., Frasquet-Deltoro, M., \& Cervera-Taulet, A. (2009). The value of B2B relationships. Industrial Management \& Data Systems, 109(5), 593-609. doi:10.1108/02635570910957605.

Grönroos, C. (1997). Value-driven relational marketing: From products to resources and competencies. Journal of marketing management, 13(5), 407-419. doi:10.1080/0267257x.1997.9964482.

Guerin, T. F. (1999). Transfer of Australian environmental research on the insecticide endosulfan to Anhui Province, China. Journal of Environmental Sciences (China), 11(4), 443-448. Id: 1001-0742(1999)04-0443-06.

Gunasekara, C. (2005). Reframing the role of universities in the development of regional innovation systems. The Journal of Technology Transfer, 31(1), 101-113. doi:10.1007/s10961-005-5016-4.

Hair, J. F., Hult, G. T. M., Ringle, C., \& Sarstedt, M. (2013). A Primer on Partial Least Squares Structural Equation Modeling (PLS-SEM). Newbury Park, CA: Sage Publications.

Hair Jr, J. F., Hult, G. T. M., Ringle, C., \& Sarstedt, M. (2016). A primer on partial least squares structural equation modeling (PLS-SEM) ( $2^{\text {nd }}$ ed.), Thousand Oaks: Sage Publications.

Helgesen, Ø. (2008). Marketing for higher education: A relationship marketing approach. Journal of Marketing for Higher Education, 18(1), 50-78. doi:10.1080/08841240802100188.

Hennig-Thurau, T., \& Klee, A. (1997). The impact of customer satisfaction and relationship quality on customer retention: A critical reassessment and model development. Psychology \& marketing, 14(8), 737-764. doi:10.1002/ (SICI)1520-6793(199712)14:8<737::AID-MAR2>3.0.CO;2-F.

Hrebiniak, L. G. (1974). Effects of job level and participation on employee attitudes and perceptions of influence. Academy of Management Journal, 17(4), 649-662. doi:10.2307/255644.

Huntley, J. K. (2006). Conceptualization and measurement of relationship quality: Linking relationship quality to actual sales and recommendation intention. Industrial Marketing Management, 35(6), 703-714. doi:10.1016/j.indmarman.2005.05.011.

Jones, T. O., \& Sasser, W. (1998). Why satisfied customers defect. IEEE Engineering Management Review, 26(3), 16-26.

Kumar, N., Scheer, L. K., \& Steenkamp, J.-B. E. (1995). The effects of supplier fairness on vulnerable resellers. Journal of marketing research, 32(1), 54-65. doi:10.2307/3152110. 
Lantos, G. P. (1994). Faculty Internships: A Means to Bridge the Academician-Practitioner Gap. Journal of Product \& Brand Management, 3(4), 15-30. doi:10.1108/10610429410073101.

Lashley, C., \& Morrison, A. (2003). Hospitality as a'commercial friendship'. Hospitality Review, 5(4), 31-36.

Lee, Y. S. (2000). The sustainability of university-industry research collaboration: an empirical assessment. The Journal of Technology Transfer, 25(2), 111-133. doi: 10.1023/a:1007895322042.

Leydesdorff, L. (2012). The Triple Helix, Quadruple Helix,..., and an N-tuple of helices: Explanatory models for analyzing the knowledge-based economy? Journal of the Knowledge Economy, 3(1), 25-35. doi:10.1007/s13132-011-0049-4.

Leydesdorff, L., \& Etzkowitz, H. (1998). Triple Helix of innovation: introduction. Science and Public Policy, 25(6), 358-364. doi:10.1093/ $\mathrm{spp} / 25.6 .358$.

Lundvall, B.-A. (1997). National systems and national styles of innovation. Paper presented at the Fourth International ASEAT Conference, "Differences in 'styles' of technological innovation," Manchester, UK.

Martinelli, A., Meyer, M., \& von Tunzelmann, N. (2008). Becoming an entrepreneurial university? A case study of knowledge exchange relationships and faculty attitudes in a medium-sized, research-oriented university. The Journal of Technology Transfer, 33(3), 259-283. doi:10.1007/s10961-007-9031-5.

Marzo-Navarro, M., Pedraja-Iglesias, M., \& Rivera-Torres, P. (2009). The marketing approach in relationships between universities and firms. Journal of Relationship Marketing, 8(2), 127-147. doi:10.1080/15332660902876869.

McDonald, G. W. (1981). Structural exchange and marital interaction. Journal of Marriage and the Family, 43(4), 825-839. doi: $10.2307 / 351340$.

Moorman, C., Zaltman, G., \& Deshpande, R. (1992). Relationships between providers and users of market research: The dynamics of trust. Journal of marketing research, 29(3), 314-328. doi: $10.2307 / 3172742$.

Mora-Valentin, E. M., Montoro-Sanchez, A., \& Guerras-Martin, L. A. (2004). Determining factors in the success of R\&D cooperative agreements between firms and research organizations. Research Policy, 33(1), 17-40. doi:10.1016/s0048-7333(03)00087-8.

Morgan, R. M., \& Hunt, S. D. (1994). The commitment-trust theory of relationship marketing. the Journal of Marketing, 58(3), 20-38. doi: $10.2307 / 1252308$.
Necoechea-Mondragón, H., Pineda-Domínguez, D., \& Soto-Flores, R. (2013). A conceptual model of technology transfer for public universities in Mexico. Journal of technology management \& innovation, 8(4), 24-35. doi: 10.4067/S0718-27242013000500003.

Newman, J. W., \& Werbel, R. A. (1973). Multivariate analysis of brand loyalty for major household appliances. Journal of marketing research, 10(4), 404-409. doi:10.2307/3149388.

Nunnally, J. C., \& Bernstein, I. H. (1994). The theory of measurement error. Psychometric theory (209-247), New York, McGraw-Hill. doi:10.1177/014662167900300216.

Oliver, R. L. (1999). Whence consumer loyalty? the Journal of Marketing, 63, 33-44. doi:10.2307/1252099.

Perkmann, M., \& Walsh, K. (2007). University-industry relationships and open innovation: Towards a research agenda. International Journal of Management Reviews, 9(4), 259-280. doi:10.1111/j.1468-2370.2007.00225.x.

Plewa, C., Quester, P., \& Baaken, T. (2005). Relationship marketing and university-industry linkages: a conceptual framework. Marketing Theory, 5(4), 433-456. doi:10.1177/1470593105058824.

Plewa, C., \& Quester, P. (2007). Key drivers of university-industry relationships: the role of organisational compatibility and personal experience. Journal of Services Marketing, 21(5), 370-382. doi:10.1108/0887604071077367.

Plewa, C., Korff, N., Johnson, C., Macpherson, G., Baaken, T., \& Rampersad, G. C. (2013). The evolution of university-industry linkagesA framework. Journal of Engineering and Technology Management, 30(1), 21-44. doi:10.1016/j.jengtecman.2012.11.005.

Price, L. L., \& Arnould, E. J. (1999). Commercial friendships: service provider-client relationships in context. Journal of Marketing, 63(4), 38-56. doi:10.2307/1251973.

Pruitt, D. G. (1981). Introduction: An overview of negotiation. Negotiation behavior (Vol. 47, pp. 1-17). New York: Academic Press. doi:10.1016/b978-0-12-566250-5.50006-9.

Razali, N. M., \& Wah, Y. B. (2011). Power comparisons of shapiro-wi$\mathrm{lk}$, kolmogorov-smirnov, lilliefors and anderson-darling tests. Journal of Statistical Modeling and Analytics, 2(1), 21-33.

Rosendo Ríos, V. (2013). Relationship marketing factors as key predictors of interfirm cooperation and success Cambridge Business \& Economics Conference.

Rotter, J. B. (1967). A new scale for the measurement of interpersonal trust1. Journal of personality, 35(4), 651-665. doi:10.1111/j.1467-6494.1967.tb01454.x. 
Sábato, J., \& Botana, N. (1968). La ciencia y la tecnología en el desarrollo futuro de América Latina. Revista de la Integración, 1(3), 15-36.

Scheaffer, R. L., Mendenhall, W., \& Ott, L. (2007). Elementos de muestreo: Editorial Paraninfo.

Schechter, L. (1984). A normative conception of value. Progressive Grocer, Executive Report, 2, 12-14.

Shapiro, S. S., \& Wilk, M. B. (1965). An analysis of variance test for normality (complete samples). Biometrika, 52(3-4), 591-611. doi:10.1093/biomet/52.3-4.591.

Setyawati, S. M., \& Raharja, M. C. (2018). Trust Dimensions Model in Creating Loyalty Stage for Service Consumers of Sharia Rural Banking. European Research Studies Journal, 21(1), 507-518.

Tax, S. S., Brown, S. W., \& Chandrashekaran, M. (1998). Customer evaluations of service complaint experiences: implications for relationship marketing. Journal of Marketing, 62(2), 60-76. doi:10.2307/1252161.

Tian, Y., Lai, F., \& Daniel, F. (2008). An examination of the nature of trust in logistics outsourcing relationship: empirical evidence from China. Industrial Management \& Data Systems, 108(3), 346-367. doi:10.1108/02635570810858769.

Ulaga, W., \& Eggert, A. (2006). Relationship value and relationship quality: Broadening the nomological network of business-to-business relationships. European Journal of Marketing, 40(3/4), 311-327. doi:10.1108/03090560610648075.
Umar, A., \& Bahrun, R. (2017). The Mediating Relationship of Customer Satisfaction Between Brand Trust, Brand Social Responsibility Image with Moderating Role of Switching Cost. Advanced Science Letters, 23(9), 9020-9025.

Voss, G. B., Parasuraman, A., \& Grewal, D. (1998). The roles of price, performance, and expectations in determining satisfaction in service exchanges. Journal of Marketing, 62(4), 46-61. doi:10.2307/1252286.

Wulf, K. D., Odekerken-Schröder, G., \& Iacobucci, D. (2001). Investments in consumer relationships: a cross-country and cross-industry exploration. Journal of marketing, 65(4), 33-50. doi:10.1509/ jmkg.65.4.33.18386.

Zacarías, M., \& Martín, H. (2011). La relación del desarrollo: universidad-gobierno-empresa. Caso comparativo México, Corea y China. Portes: Revista Mexicana de Estudios Sobre la Cuenca del Pacifico, $5(10)$.

Zeithaml, V. A. (1988). Consumer perceptions of price, quality, and value: a means-end model and synthesis of evidence. Journal of Marketing, 52(3), 2-22. doi:10.2307/1251446.

Zeithaml, V. A., Berry, L. L., \& Parasuraman, A. (1996). The behavioral consequences of service quality. Journal of Marketing, 60(2), 31-46. doi:10.2307/1251929. 
